\title{
Focused ultrasound: crossing barriers to treat Alzheimer's disease
}

\begin{abstract}
"Focused ultrasound will improve the capacity for several drug categories to pass the blood-brain barrier ... which may be curative in addition to providing symptomatic relief for Alzheimer's disease patients."
\end{abstract}

Keywords: Alzheimer's disease $\boldsymbol{\square}$ blood-brain barrier $\boldsymbol{\|}$ drug delivery $\boldsymbol{\square}$ focused ultrasound $\boldsymbol{\square}$ microbubbles

Alzheimer's disease (AD) is the leading cause of dementia in the elderly and the seventh leading cause of death in North America [101]. It is a progressive and irreversible neurodegenerative disease that has no cure. At the molecular and cellular levels, $\mathrm{AD}$ is characterized by the presence of plaques composed of amyloid- $\beta$ (A $\beta$ ) peptides, neurofibrillary tangles, neuronal loss and deficits in neurotransmitters [1]. In people living with $\mathrm{AD}$, this pathology translates into symptoms such as a decline in reasoning, loss of memory and general deterioration of cognitive capacities. Current AD treatments are limited to drugs that improve neurotransmitter function and provide symptomatic relief [2]. The development of novel therapeutics for curing $A D$ focuses on either preventing amyloid aggregation or initiating break-up of amyloid plaques [1]. A limiting factor in targeting the brain with novel therapeutics is the presence of the blood-brain barrier (BBB) [3]. The BBB is a specialized structure that restricts passage of molecules, including most drugs, from the blood into the brain, presenting a challenge for the pharmaceutical treatment of brain diseases.

Regarding the delivery of therapeutics to eradicate $\mathrm{AD}$ pathology, technological advances in the field of focused ultrasound (FUS) hold great promise to bypass the $\mathrm{BBB}$ and target the brain without involving surgical approaches. It is now established that transcranial FUS can increase the permeability of the BBB and allow the passage of molecules from the blood to the brain in an efficient manner $[4,5]$. The process of FUS concentrates acoustic energy and deposits it in a small target volume in the body with minimal or no consequences to the surrounding tissue. There are two major effects, thermal and nonthermal, which can be observed at the target. For thermal applications, the ultrasound energy is converted to heat, which can be used to activate heat-sensitive drugs or to ablate tumors at localized targets [6]. For nonthermal applications, the ultrasound energy induces cavitation (oscillation of the bubbles in the tissue), which produces mechanical changes in the tissue [7]. Over the last 10 years, it has been demonstrated that FUS energy combined with microbubble contrast agents can be used to increase the permeability of the BBB, temporarily and reversibly, to allow access of therapeutic agents to the brain [8]. These findings provide a major breakthrough for treatment of brain diseases such as AD.

\section{Targeting the brain with FUS: overcoming the skull challenge}

Delivery of therapeutics to the brain using FUS has been complicated by the skull, which strongly attenuates energy and causes distortion of the ultrasound field. Therefore, it was long believed that FUS would have limited relevance for the treatment of brain diseases [9]. Early work by the Fry brothers demonstrated that FUS could induce changes in the brain, but at that time the technique was only possible after a craniotomy [10]. Recent production of phased-array transducers to distribute energy over the entire skull surface, in combination with high-performance computers to correct ultrasound wave distortions caused by the skull, has made transcranial therapy possible [11]. Similarly, improvements in the ability to target small, specific structures with ultrasound have been addressed by combining FUS with guidance by MRI (termed MRIgFUS) [12,13]. This significant progress in the field of transcranial FUS led to a clinical trial for the treatment of glioblastoma [14] and chronic pain [15], and to intense research aimed at promoting drug delivery to the brain by transiently and reversibly opening the BBB $[5,7,8]$.

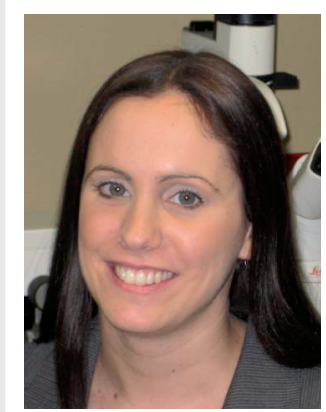

Alison Burgess

Imaging Research, Sunnybrook Research Institute, ON, Canada

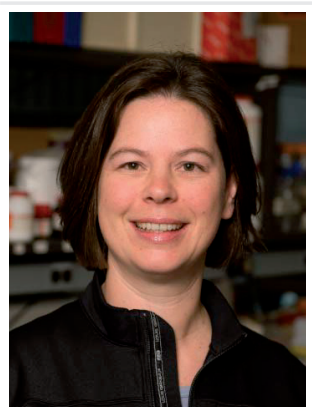

Isabelle Aubert

Brain Sciences, Sunnybrook Research Institute, Toronto, ON,

Canada, and,

Laboratory Medicine \&

Pathobiology, University of

Toronto, Toronto, ON, Canada

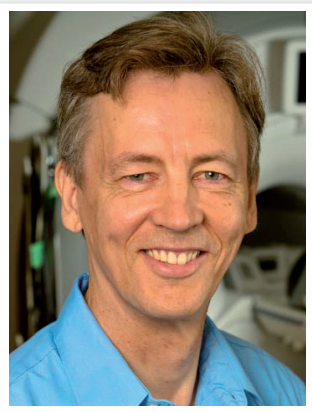

Kullervo Hynynent

Imaging Research, Sunnybrook Research Institute, 2075 Bayview Avenue, S665 Toronto, ON, Canada, M4N 3M5, and,

Medical Biophysics, University of Toronto, Toronto, ON, Canada ${ }^{\dagger}$ Author for correspondence: Tel.: + I 4164805717

E-mail: kullervo.hynynen@ sunnybrook.ca

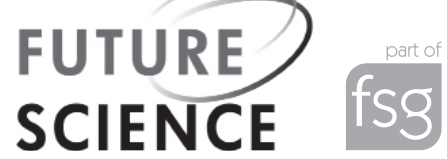




\section{The power of microbubbles}

Stable microbubbles have been used as contrast agents for medical imaging for decades, but the use of microbubbles for $\mathrm{BBB}$ disruption with ultrasound is a relatively recent discovery [4]. Prior to the use of microbubbles, FUS-induced BBB opening was almost always associated with hemorrhage and tissue damage due to the induction of unpredictable, inertial cavitation [16-18]. It was hypothesized that the preformed microbubbles injected into the bloodstream would act as energy-concentrators when exposed to ultrasound and would expand and contract at the frequency of the propagating acoustic wave, leading to BBB disruption [4]. Indeed, it was found that the presence of microbubbles in the bloodstream allows the BBB to be opened using a significantly reduced acoustic power, over 100-times less than that required to produce thermal damage in the tissue [8]. Recently, a passive cavitation detector was used to verify that a FUS-disrupted BBB was probably due to oscillations of microbubbles with the blood vessel, but was not dependent on violent collapse of bubbles (inertial cavitation), which is often associated with tissue damage $[19,20]$. Studies using electron microscopy have identified four potential methods of transport of circulating molecules across the BBB after transcranial FUS with microbubbes: transcytosis via endothelial cell receptors, between endothelial cells following mechanical disruption of the tight junctions, through the endothelial cells themselves by opening of the cell cytoplasm and by way of injury to the endothelial lining [21,22]. Regardless of the mechanism of action, it has been repeatedly observed that enhanced permeability of the BBB by FUS and microbubbles is safe and transient. Contrast-enhanced MRI has demonstrated that the efficacy of the BBB is restored at $6 \mathrm{~h}$ following disruption, and the BBB remained impenetrable 2 days, 5 days and 4 weeks later [23,24].

\section{The FUS advantage for BBB opening}

$A$ variety of strategies have been used to improve distribution of therapeutics to the brain. Direct delivery into the brain via surgery or through an implanted device can result in targeted delivery, but these surgical procedures carry severe risks. The systemic administration of osmotic solutions [24], mannitol [25] and various chemicals including vasodilators [26] and solvents [27,28], have also been used to increase BBB permeability, but many of these agents are toxic and can cause neuronal damage. Since the incorporation of microbubbles into the FUS procedure, there has been no evidence of ischemic or apoptotic regions after BBB disruption $[23,29,30]$. Overall, the effects to the brain from FUS appear minimal and certainly less than what has been observed from other methods for drug delivery to the brain. In addition, systemic administration of chemical agents results in widespread permeabilization of the BBB. Such extensive opening of the BBB can have serious side effects since the drugs and other potentially cytotoxic compounds present in the blood will have direct access to the entire CNS for long periods of time. These side effects are eliminated with MRIgFUS as the BBB opening is carefully controlled; for example, the BBB could be opened in a small region of the substantia nigra or the striatum, which may be used to improve drug delivery for Parkinson's disease without affecting the rest of the brain. Alternatively, in the case of $\mathrm{AD}$ where pathology is extensive and widespread disruption of the BBB is desirable, the selectivity of MRIgFUS still presents an advantage over indiscriminate chemical agents. FUS allows the BBB to be disrupted sequentially over a large timescale: one brain region is allowed to recover prior to the disruption of the next region. In conjunction with the briefness with which the BBB is disrupted with FUS, this sequential approach is able to treat the entire brain with minimal exposure to other potentially toxic blood components.

\section{"...the effects to the brain from focused ultrasound appear minimal and certainly less than what has been observed from other methods for drug delivery to the brain."}

Currently, systemic administration of therapeutics for treatment of neurodegenerative disease involves multiple doses of high concentrations of drug, delivered systematically over several months or years [31]. FUS can be used to overcome the low penetration of drugs into the brain and the unwanted side effects associated with high levels of circulating drugs by increasing the permeability of the BBB. Using FUS to improve drug penetration will also decrease the cost of the therapy and reduce the number of injections, making treatments more practical and easier on the patients and their caregivers.

Drug delivery using FUS is not limited to treatment of neurodegenerative disease. The disruption of the BBB using FUS has many applications in the brain, such as improving access of chemotherapy agents to brain tumors, targeting gene therapy and delivering antibodies for breast cancer metastases [32]. 


\section{The potential of FUS to improve AD therapies}

Immunotherapy with $A \beta$ is based on the ability of $A \beta$ antibodies to clear plaques from the brain and decrease toxicity to remaining neuronal populations. In mouse models of $\mathrm{AD}$, systemic administration of $A \beta$ antibodies causes a marked reduction in amyloid burden and an improvement in cognitive function [33]. Recently, it was shown that direct delivery of low doses of $A \beta$ antibodies to the brain via intracranial injection was more efficient at reducing $A \beta$ plaques than peripheral injections of high doses of antibody [34,35]. Furthermore, the directdelivery method minimized the unwanted side effects of excess antibody on the vasculature [34]. Despite the efficiency of the treatment, intracranial injections represent obvious challenges for translational application in $\mathrm{AD}$ patients. The use of FUS to open the BBB offers the potential to benefit from the increased efficiency and efficacy of direct drug injection without the need for an invasive surgery.

"Focused ultrasound can be used to overcome the low penetration of drugs into the brain and the unwanted side effects associated with high

levels of circulating drugs by increasing the permeability of the blood-brain barrier."

The first evidence that FUS may be applicable for delivery of antibodies to the brain came in 2006 when Kinoshita and colleagues demonstrated that antibodies circulating in the bloodstream can be detected in the brain after BBB opening by FUS in young mice [36,37]. Despite these findings, administration of antibodies to $\mathrm{AD}$ brains was uncertain since the amyloid pathology, which is present in the brain and the vasculature, is suggested to significantly impact the properties and function of the BBB [38]. Initial studies were aimed at addressing the safety of opening the $\mathrm{BBB}$ in $\mathrm{AD}$ mice. The results demonstrated that the properties of $\mathrm{BBB}$ disruption by transcranial FUS were not significantly different in $\mathrm{AD}$ transgenic mice (PS1/APP), compared with wild-type controls [39]. Furthermore, in a similar AD mouse model (PDAPP), it was found that the opening of the $\mathrm{BBB}$ was comparable in aged mice (12 vs 26 months), despite an increased brittleness of the skull and altered vasculature observed in the older transgenic animals [40]. Raymond and colleagues also showed that $A \beta$ antibodies given in the bloodstream were co-localized with trypan blue-stained plaques in the brain following BBB opening with FUS [40]. These studies demonstrated the feasibility of targeting amyloid plaques present in the brain with $A \beta$ antibodies administered in the bloodstream that crosses the BBB after transcranial FUS.

\section{"The use of focused ultrasound to open the blood-brain barrier offers the potential to benefit from the increased efficiency and efficacy of direct drug injection without the need for an invasive surgery."}

The landmark study demonstrating the efficacy of FUS in reducing amyloid plaque pathology was published in 2010 [41]. TgCRND mice that develop extensive amyloid deposits and display cognitive deficits were used in this study. A total of $40 \mu \mathrm{g}$ of BAM-10 (anti-A $\beta$ ) antibodies were injected into the circulation through a tail-vein catheter. Immediately after this, the BBB was opened at four locations in the right hemisphere using a custom-built FUS system. At all time points after BBB opening ( 4 h, 2 and 4 days), BAM-10 antibody was found to specifically bind to plaques only on the right, MRIgFUS-targeted side of the brain. No BAM-10 antibody was detected in the left hemisphere, indicating the antibody could be delivered from the peripheral circulation to targeted areas of the brain. Stereology was performed 4 days after treatment and revealed that there were $12 \%$ fewer plaques in the cortex on the MRIgFUS-targeted side compared with the control side. In addition, the mean plaque size was reduced by $12 \%$ and the surface area of the plaques was reduced by $23 \%$, indicating significant overall reduction in AD pathology. This study is the first evidence that FUS can be used to target drug delivery and reduce AD pathology.

Clinical trials using $A \beta$ antibodies in $A D$ patients have entered Phase III with hopes of success somewhat dampened by cautionary notes on safety and efficacy $[42,43]$. Bapineuzumap, a fully humanized $A \beta$ antibody, raised safety concerns after 12 of 238 patients developed vasogenic edema [31]. These results highlight the potential human safety issues associated with peripheral $A \beta$ clearance. In our opinion, targeting the delivery of the antibody to the brain could benefit the passive-immunization approach to $\mathrm{AD}$ by reducing the levels of circulating antibody and by improving the penetration of the antibody to the brain regions where it is needed most. 


\section{The future of FUS in the treatment of $A D$}

Bowman et al., suggested that the presence of amyloid in the AD brain can impair the structure and function of the $\mathrm{BBB}$ and that increased degrees of BBB dysfunction are associated with increased rates of neurodegeneration [44]. The status of the $\mathrm{BBB}$ in $\mathrm{AD}$ is variable between patients, but it remains clear that drug delivery to the brain in AD could be improved by FUS. Importantly, the BBB seems able to restore its integrity even under deleterious circumstances. For example, following a stroke or brain trauma, physical damage to the blood vessels at the site of injury temporarily disrupts normal $\mathrm{BBB}$ function and allows compounds present in the blood to gain direct access to brain tissue. Still, the BBB can return to normal function after only hours [45]. The plasticity of the BBB in other damaging conditions as well as some preliminary evidence $[39,40]$ suggests that even in the AD brain, the BBB could sustain some disruption with MRIgFUS and be capable of restoring its integrity and function over time. Careful evaluation of the effect of FUS on the $\mathrm{BBB}$ in mouse models of $\mathrm{AD}$ is underway.

Using FUS to open the BBB will significantly broaden the type of therapeutics being developed for treatment of AD. FUS will improve the capacity for several drug categories to pass the $\mathrm{BBB}$, related to the amyloid cascade and directed onto novel targets, which may be curative in addition to providing symptomatic relief for AD patients.

\section{"The plasticity of the blood-brain barrier in other damaging conditions ... suggests that even in the Alzheimer's disease brain, the blood-brain barrier could sustain some disruption with MRI-guided focused ultrasound and be capable of restoring its integrity and function over time."}

The transient opening of the BBB by FUS has therapeutic implications that extend beyond traditional drug delivery to the brain. Cell replacement and gene therapy to the brain and spinal cord could be envisioned using MRIgFUS. Experiments are necessary to determine whether FUS can open the BBB enough to allow cell entry and to facilitate gene delivery to the brain without causing damage to the tissue.

Finally, the use of FUS could potentially increase neuronal activity and perhaps be of benefit by targeting specific neuronal pathways in $\mathrm{AD}$ and in other neurodegenerative disorders. This type of research is in its infancy and much remains to be understood on the effects of FUS to the brain, but some studies have shown promise for the use of FUS to stimulate neuronal activity. Indeed, low-frequency ultrasound can activate sodium- and calcium-gated channels leading to increased neuronal activity [46]. Furthermore, Tufail and colleagues demonstrated that ultrasound stimulation could increase the electrical activity of neurons in the motor cortex without inducing $\mathrm{BBB}$ opening or causing any damage to the tissue [47]. The authors also found that FUS stimulation of the hippocampus, a structure relevant to learning and memory and which is affected in AD, increased electrical activity and the levels of brain-derived neurotrophic factor, an important regulator of memory consolidation. These studies raise the interesting possibility that FUS alone may be able to stimulate brain circuits and enhance levels of neurotrophins and neurotransmitter release, all of which could have beneficial effects on the AD brain.

\section{Conclusions}

Focused ultrasound with microbubbles holds great promise for targeted delivery of therapeutics to treat neurodegenerative diseases such as AD. It has already been demonstrated that FUS improves delivery of $A \beta$ antibodies to the brain, resulting in decreased amyloid pathology in a mouse model of AD. The effects of FUS on the brain and optimal antibody delivery need to be further evaluated to ensure the safety of the procedure before human patients are treated. However, slight modifications of the technology currently tested in clinical trials for thermal ablation of tumors could make MRIgFUS applications to the AD brain possible. Targeting specific brain regions of interest with MRIgFUS holds great promise for the development of treatments for AD and other brain disorders.

\section{Financial \& competing interests disclosure}

Kullervo Hynynen is an investor in patents related to focusing ultrasound through skull and disrupting the blood-brain barrier with ultrasound. These patents are owned by Brigham and Women's Hospital, Boston, MA, USA. The authors have no other relevant affliations or financial involvement with any organization or entity with a financial interest in or financial conflict with the subject matter or materials discussed in the manuscript apart from those disclosed.

No writing assistance was utilized in the production of this manuscript. 


\section{Bibliography}

Papers of special note have been highlighted as:

- of interest

-" of considerable interest

1 Carter MD, Simms GA, Weaver DF. The development of new therapeutics for Alzheimer's disease. Clin. Pharmacol. Ther. 88, 475-486 (2010).

2 Massoud F, Gauthier S. Update on the pharmacological treatment of Alzheimer's disease. Curr. Neuropharmacol. 8, 69-80 (2010).

3 Pardridge WM. Alzheimer's disease drug development and the problem of the blood-brain barrier. Alzheimers Dement. 5 , 427-432 (2009).

4 Hynynen K, McDannold N, Vykhodtseva N, Joelsz FA. Noninvasive MR imaging-guided focal opening of the blood-brain barrier in rabbits. Radiology 220, 640-646 (2001).

-. The first study to document reliable blood-brain barrier (BBB) opening with focused ultrasound (FUS) in the presence of microbubble contrast agents. MRI and histology were used to demonstrate the safety of the procedure.

5 Vykhodtseva N, McDannold N, Hynynen K. Progress and problems for the application of focused ultrasound for blood-brain barrier disruption. Ultrasonics 48, 279-296 (2008).

6 Hynynen K, McDannold N. MRI guided and monitored focused ultrasound thermal ablation methods: a review of progress. Int. J. Hyperthermia 20, 725-737 (2004).

7 Hernot S, Klibanov AL. Microbubbles in ultrasound-triggered drug and gene delivery. Adv. Drug Deliv. Rev. 60, 1153-1166 (2008).

8 Hynynen K. Ultrasound for drug and gene delivery to the brain. Adv. Drug Deliv. Rev. 60, 1209-1217 (2008).

9 Lynn JG, Zwemer RL, Chick AJ, Miller AE. A new method for the generation and use of focused ultrasound in experimental biology. J. Gen. Physiol. 26, 179-193 (1942).

10 Fry WJ, Mosberg WH, Barnard JW, Fry FJ. Production of focal destructive lesions in the central nervous system with ultrasound. J. Neurosurg. 11, 471-478 (1954).

11 Hynynen K, Clement G. Clinical applications of focused ultrasound - the brain. Int. J Hypotehrmia 23, 193-202 (2007).

12 Hynynen K, Darkazanli A, Unger E, Schenck JF. MRI-guided noninvasive ultrasound surgery. Med. Phys. 20, 107-115 (1993).
13 Cline HE. Hynynen K, Hardy CJ, Watkins RD, Schenck FJ, Jolesz FA. MR temperature mapping of focused ultrasound surgery. Magn. Reson. Med. 30, 98-106 (1994).

14 McDannold N, Clement GT, Black P, Jolesz F, Hynynen K. Transcranial magnetic resonance imaging-guided focused ultrasound surgery of brain tumors: initial findings in 3 patients. Neurosurg. 66, 323-332 (2010).

15 Martin E, Jeanmonod D, Morel A, Zadicario E, Werner B. High-intensity focused ultrasound for noninvasive functional neurosurgery. Ann. Neurol. 66, 858-861 (2009).

16 Vykhodtseva N. Effects of high intensity pulsed ultrasound on brain tissues. Proceedings of: The 5th International Symposium on Ultrasound in Biological Medicine. Puschino, Russia 7-11 September 1981.

17 McDannold N, Vykhodtseva N, Jolesz FA. MRI investigation of the threshold for thermally induced blood-brain barrier disruption and brain-tissue damage in the rabbit brain. Magn. Reson. Med. 51, 913-923 (2004).

18 Mesiwala AH, Farrell L, Wenzel HJ et al. High-intensity focused ultrasound selectively disrupts the blood-brain barrier in vivo. Ultrasound Med. Biol. 28, 389-400 (2004).

19 McDannold N, Vykhodtseva N, Hynynen K. Targeted disruption of the blood-brain barrier with focused ultrasound: associated with cavitation activity. Phys. Med. Biol. 51, 793-807 (2006).

20 Tung YS, Vlachos F, Choi JJ, Deffieux T, Selert K, Jonofagou EE. In vivo transcranial cavitation threshold detection during ultrasound-induced blood-brain barrier opening in mice. Phys. Med. Biol. 55, 6141-6155 (2010).

21 Sheikov N, McDannold N, Sharma S, Hynynen K. Effect of focused ultrasound applied with an ultrasound contrast agent on the tight junctional integrity of the brain microvascular endothelium. Ultrasound Med. Biol. 34, 1093-1104 (2008).

22 Sheikov N, McDannold N, Vykhodtseva N, Jolesz F, Hynynen K. Cellular mechanisms of the blood-brain barrier opening induced by ultrasound in the presence of microbubbles. Ultrasound Med. Biol. 30, 979-989 (2004).

- The authors use electron microscopy to identify four possible cellular mechanisms of transcapillary passage during BBB disruption by FUS.

23 McDannold N, Vykhodtseva N, Raymond S, Joelsz FA, Hynynen K. MRI-guided targeted blood-brain barrier disruption with focused ultrasound: histological findings in rabbits. Ultrasound Med. Biol. 31, 1527-1537 (2005).

24 Rapoport SI. Advances in osmotic opening of the blood-brain barrier to enhance CNS chemotherapy. Expert Opin. Investig. Drugs 10, 1809-1818 (2001).

25 Zunkeler B, Carson RE, Olson J, Blasberg RG, DeVroom H, Lutz RJ. Quantification and pharmacokinetics of blood-brain barrier disruption in humans. J. Neurosurg. 85, 1056-1065 (1996).

26 Matsukado K, Sugita M, Black KL. Intracarotid lose dose bradykinin infusion selectively increases tumor permeability through activation of B2 receptors in malignant gliomas. Brain Res. 792, 10-15 (1998).

27 Hanig JP, Morrison JM Jr, Krop S. Ethanol enhancement of blood brain barrier permeability to catecholamines in chicks. Eur. J. Pharmacol. 18, 79-82 (1972).

28 Broadwell RD, Salcman M, Kaplan RS. Morphologic effect of dimethyl sulfoxide on the blood-brain barrier. Science 217, 164-166 (1982).

29 Hynynen K, McDannold N, Sheikov NA, Jolesz FA, Vykhodtseva N. Local and reversible blood-brain barrier disruption by noninvasive focused ultrasound at frequencies suitable for trans-skull sonciations. NeuroImage 24, 12-20 (2005).

30 Hynynen K, McDannold N, Vykhodtseva N et al. Focal disruption of the blood-brain barrier due to $260-\mathrm{kHz}$ ultrasound bursts: a method for molecular imaging and targeted drug delivery. J Neurosurg. 105, 445-454 (2006).

31 Panza F, Frisardi V, Imbimbo BP et al. Bapineuzumab: anti- $\beta$-amyloid monoclonal antibodies for the treatment of Alzheimer's disease. Immunotherapy 2, 767-782 (2010).

32 Hynynen K. MRI-guided focused ultrasound treatments. Ultrasonic 50, 221-229 (2010).

33 Bard F, Cannon C, Barbour R et al. Peripherally administered antibodies against amyloid $\beta$-peptideenter the central nervous system and reduce pathology in a mouse model of Alzheimer's disease. Nat. Med. 6, 916-919 (2000).

34 Thakker DR, Weatherspoon MR, Harrison J, Keene TE, Lane DS. Intracerebroventricular amyloid- $\beta$ antibodies reduce cerebral amyloid angiopathy and associated micro-hemorrhages in aged Tg2576 mice. Proc. Natl Acad. Sci. USA 106, 4501-4506 (2009).

- Thakker and colleagues demonstrate the potential for locally administered amyloid immunotherapy to reduce brain pathology 
of Alzheimer's disease (AD) and also decrease the corresponding cerebral amyloid angiopathy. This study suggests that localized passive immunization holds great promise for the treatment of AD.

35 Wilcock DM, DiCarlo G, Henderson D, Jackson J, Clarke K. Intracranially administered anti-A $\beta$ antibodies reduce $\beta$-amyloid deposition by mechanisms both independent of and associated with microglial activation. J. Neurosci. 23, 3745-3751 (2003).

36 Kinoshita M, McDannold N, Jolesz FA, Hynynen K. Targeted delivery of antibodies through the blood-brain barrier by MRI-guided focused ultrasound. Biochem. Biophys. Res. Commun. 340, 1085-1090 (2006).

37 Kinoshita M, McDannold N, Jolesz FA, Hynynen K. Noninvasive localized delivery of Herceptin to the mouse brain by MRI-guided focused ultrasound-induced blood-brain barrier disruption. Proc. Natl Acad. Sci. USA 103, 11719-11723 (2006).

38 Weller RO, Preston SD, Subash M, Carare RO. Cerebral amyloid angiopathy in the aetiology and immunotherapy of Alzheimer's disease. Alzheimers Res. Ther. 1, 6-19 (2009).

39 Choi JJ, Wang S, Brown TR, Small SA, Duff KE, Konofagou EE. Noninvasive and transient blood-brain barrier opening in the hippocampus of Alzheimer's double transgenic mice using focused ultrasound. Ultrasonic Imaging 30, 189-200 (2008).

40 Raymond SB, Treat LH, Dewey JD, McDannold NJ, Hynynen K, Bacskai BJ. Ultrasound enhanced delivery of molecular imaging and therapeutic agents in Alzheimer's disease mouse models. PLoS One 3, 1-7 (2008).

- The authors were the first to disrupt the $\mathrm{BBB}$ in transgenic mouse models of $\mathrm{AD}$, thereby demonstrating the potential for localized delivery of therapeutics to the amyloid plaques.

41 Jordāo JF, Ayala-Grosso CA, Markham K et al. Antibodies targeted to the brain with image-guided focused ultrasound reduces amyloid- $\beta$ plaque load in the TgCRND8 mouse model of Alzheimer's disease. PLoS One 5, 1-8 (2010).

- This landmark study was the first to illustrate that antibodies were concentrated in the brain following FUS-induced BBB disruption. After 7 days, the corresponding decrease in plaque pathology realizes the potential of this treatment.

42 Holtzman DM. Alzheimer's disease: moving towards a vaccine. Nature $454,418-420$ (2008).
43 Gura T. Hope in Alzheimer's fight emerges from unexpected places. Nat. Med. 14, 894 (2008).

44 Bowman ND, Kaye MD, Moore BS, Waichunas BA, Carlson NE, Quinn JF. Blood-brain barrier impairment in Alzheimer's disease: stability and functional significance. Neurology 68, 1809-1814 (2007).

45 Lo EH, Singhal AB, Torchilin VP. Drug delivery to the damaged brain. Brain Res. Rev. 38, 140-148 (2001).

46 Tyler WJ, Tufail Y, Finsterwald M, Tauchmann ML, Olson EJ, Majestic C. Remote excitation of neuronal circuits using low-intensity, low frequency ultrasound. PLoS One 3, 1-11 (2008).

47 Tufail Y, Matyushov A, Baldwin N et al. Transcranial pulsed ultrasound stimulates intact brain circuits. Neuron. 66, 681-694 (2010).

\section{Website}

101 Alzheimer's Association. 2010 Alzheimer's Disease Facts and Figures. www.alz.org/documents_custom/report_ alzfactsfigures2010.pdf 